\title{
Efforts to maximize the potential of CAR-T therapy for cancer, from T-bodies to CAR-immune cells
}

\author{
Hiroshi Fujiwara' ${ }^{1}$
}

Received: 18 August 2021 / Revised: 18 August 2021 / Accepted: 27 August 2021 / Published online: 13 September 2021

(C) Japanese Society of Hematology 2021

\section{Introduction}

The scientific concept of genetically endowing T cells with target-specific tumoricidal activity using antibody-mediated antigen recognition was first described by Dr. Eshhar around 1990 [1]. Such engineered T cells, which were originally called "T-bodies", are now called chimeric antigen receptor (CAR) gene-modified T cells (CAR-T). Therapy using these cells has emerged as the mainstay of modern anticancer immunotherapy, the most prominent example of which being CD19 CAR-T for relapsed/refractory B-cell malignancies [2]. As an extension of this strategy, continuous efforts have been made to expand the scope of indications to not only solid cancers, but also viral diseases such as COVID-19 [3].

\section{Overview of the current state of CAR-T therapy}

Now that clinical experience with CD19 CAR-T therapy has accumulated, several limitations of even this elaborate livecell drug have become apparent [4]. The first limitation are issues related to the biological properties of CAR-T itself, which include loss of function due to insufficient persistence, ineffective tumor trafficking, exhaustion within the immunosuppressive tumor microenvironment, and on-target/ off-tumor adverse events being inevitable due to a quite limited number of ideal therapeutic antigens. The second limitation are treatment-related systemic adverse events unique to CAR-T therapy, prominent examples of which are cytokine release syndrome (CRS) and immune effector cell-associated

Hiroshi Fujiwara

rieyunahiroshi@med.mie-u.ac.jp

1 Department of Personalized Cancer Immunotherapy, Mie University Graduate School of Medicine, 1577 Kurimamachiya-cho, Tsu City, Mie 514-8507, Japan neurotoxicity syndrome (ICANS). The third limitation are clonal disease progression and relapse mediated by cancer clones that lost the target antigen via cancer immune-editing $[5,6]$. This issue in Progress in Hematology (PIH) begins with an article by Prof. J. Joseph Melenhorst and his colleagues at Philadelphia University, the world-leading institute in this field, which elegantly reviews current drawbacks of CAR-T therapy and attempts to overcome them, particularly focusing on development of next-generation CAR-T technology, based on the perspective they have gained from their outstanding experience in CD19 CAR-T therapy.

\section{Efforts to improve CAR-T, with a focus on selection of immune cells for "off-the-shelf" therapy}

Thus far, efforts to improve CAR-T have focused intensely on optimization of the CAR gene construct [7]. However, with the recent rapid progress in immunology, molecular biology and synthetic biology, immune cells other than conventional $(\alpha \beta-)$ T cell for CAR gene-modification and other factors, such as combination with checkpoint inhibitors and small molecules for epigenetic modification and shifting of metabolic propensity, have emerged as another option besides CAR gene construct optimization for achievement of advanced CAR-T/CAR-immune cells with increased tumoricidal activity, prolonged persistence in vivo, resistance to exhaustion in the immunosuppressive tumor microenvironment (TME), greater trafficking to cancerous tissue, and greater safety (Fig. 1) [8, 9].

In consideration of this shift, this issue of PIH has particularly highlighted selection of immune cells to which the CAR gene is transduced, rather than molecular technologies in CAR gene construction. Technological advancements to expand the scope of immune cells for CAR gene engineering has simultaneously opened the door to "off-the-shelf" 

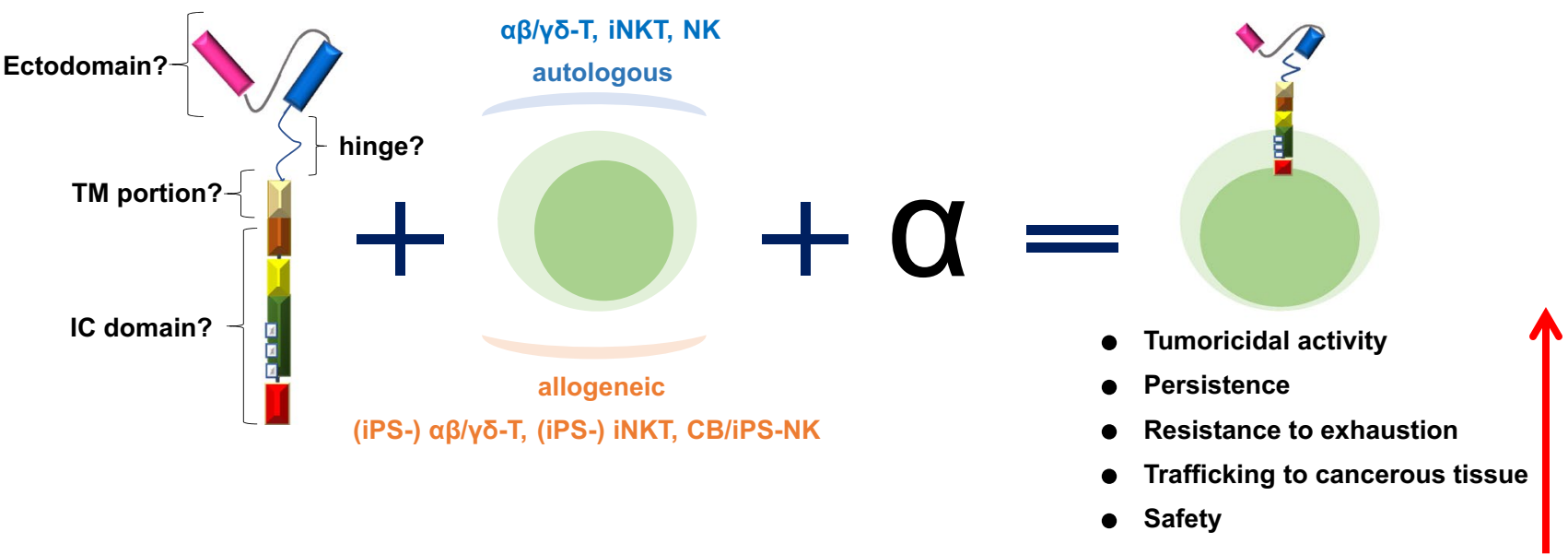

Fig. 1 Three components needed to generate advanced CAR-effector cells. Details are shown in the text. $T M$ transmembrane, $I C$ intracellular, $C B$ cord blood, $i P S$ induced pluripotent stem cell

products [9], which undoubtedly will extend clinical accessibility of this treatment strategy to the not insignificant number of cancer patients who are unfortunately less blessed with the privilege of modern cancer treatments.

In this context, this PIH has welcomed three more leading scientists/hematologists who have been successfully developing unique CAR-T cells with different immune cells.

- Adoptive therapy with CAR gene-modified CMV-specific memory $\mathrm{T}$ cells

Professor Ryotaro Nakamura and his colleagues at City of Hope Comprehensive Cancer Center has proposed clinical trials using CD19 CAR gene-transduced cytomegalovirus (CMV) reactive allogeneic memory $\mathrm{T}$ cells that uniquely display dual specificity towards CD19-positive B-lymphoid cancer cells and CMV-infected cells. This approach provides three major advantages: (1) prolonged persistence of CAR-T due to the immunological properties of CMVspecific central memory and/or memory stem T cells, (2) further expandability in vivo by patients' own circulating CMV-infected cells and exogenous CMV vaccination, and (3) expanded availability of donors who can generate this type of CAR-T bearing endogenous $T$ cell receptor with predefined target specificity, which are known to reduce risk of GVHD. Details can be found in the text.

- CAR NK cell therapy

Professor Katayoun Rezvani and her colleagues at MD Anderson Cancer Center have beautifully reviewed the current epoch-making CAR gene-modified allogeneic natural killer (NK) cell therapy, with discussion of their successful clinical trial using CD19 CAR gene-modified cord blood (CB)-derived allogeneic NK cells. Unlike allogeneic conventional $(\alpha \beta-)$ T cells, allogeneic NK cells, which are involved in innate immunity, are immunologically free from the risk of GVHD. Another advantage that NK cells offer is that they inherently provide multiple mechanisms for cytocidal activity against virus-infected cells and cancer cells [10]. A major long-unresolved issue in adoptive NK cell therapy is the short lifespan of ex vivo-expanded mature NK cells infused in vivo [10]. The authors successfully addressed this issue by establishing a large-scale purified NK cell expansion system from allogeneic CB and a novel CD19 CAR gene capable of releasing self-supporting cytokine interleukin-15. The authors also forecast the development of "off-the-shelf" CAR CB-NK cells. We believe this review will reaffirm for readers that basic science and clinical trials are much like two wheels of the same axle when it comes to development of novel treatment strategies. Details can be found in the text.

- iPS-derived immune cell-based adoptive therapy

Professor Shin Kaneko and his colleague at Kyoto University, the world-leading institute in induced pluripotent stem cell (iPS) technology, present a comprehensive review of the current state of iPS-derived immune cell-based adoptive therapy using their CAR gene-modified allogeneic iPSNK cells for solid cancers. They also discuss how iPS technology reasonably appears to have a great advantage as an 
"off-the-shelf" cellular product for adoptive immunotherapy [11]. Intensive efforts have been made to develop precedent CAR gene-modified allogeneic iPS- $\alpha \beta$-T cells, but perhaps due to the impact of clinical advances in adoptive allogeneic CAR NK therapy and the simplicity of required gene modifications for safety employment of allogeneic NK cells rather than allogeneic $\alpha \beta-T$ cells, allogeneic CAR iPS-NK cells have gained international attention as well. This systematic review will show readers how iPS-derived immune cellbased adoptive therapy is making steady progress towards clinical application.

\section{Summary}

As of the middle of 2021, COVID-19 is still wreaking havoc world-wide, including in Japan, and almost eighteen months have already passed since the first CD19 CAR-T product was approved in Japan. Three kinds of CAR-T product are currently available for routine use, and CAR-T therapy is becoming more familiar to hematologists. In that sense, now is a good time to review the current state of CAR-T therapy.

In this issue of PIH, besides providing a general overview, we focus on next-generation CAR-T using immune cells selected for particular characteristics (CAR-immune cells), which have the potential to be "off-the-shelf" products. Current trends in the development of CAR-T therapy can be largely grouped into (1) improving the clinical efficacy of validated CD19 CAR-T, and (2) expanding indications, especially to solid cancers. The important keyword for both of these trends is "off-the-shelf", which is the ideal format for practical medicine.

In this issue of PIH, all authors are world-leading researchers in this field, who have not only clinical knowledge but also detailed scientific knowledge of CAR$\mathrm{T}$. We believe this issue will strongly encourage readers to learn the modern concept of CAR-T.

\section{References}

1. Eshhar Z. Tumor-specific T-bodies: towards clinical application. Cancer Immunnol Immunother. 1997;45:131-6.

2. Frigault MJ, Maus MV. State of the art in CAR T cell therapy for CD19+ B cell malignancies. J Clin Invest. 2020;130(4):1586-94.

3. Ma M, Badeti S, Geng K, Liu D. Efficacy of targeting SARSCoV-2 by CAR-NK cells. 2020. bioRxiv. 2020.08.11.247320.

4. June CH, Sadelain M. Chimeric antigen receptor therapy. N Engl J Med. 2018;379(1):64-73.

5. Schreiber RD, Old LJ, Smyth MK. Cancer immunoediting; integrating immunity's roles in cancer suppression and promotion. Science. 2011;331(6024):1565-70.

6. Fry TJ, Shah NN, Orentas RJ, et al. CD22-targeted CAR T cells induce remission in B-ALL that is naïve or resistant to CD19targeted CAR immunotherapy. Nat Med. 2018;24(1):20-8.

7. Rafiq S, Hackett SR, Brentjens RJ. Engineering strategies to overcome the current roadblocks in CAR T cell therapy. Nat Rev Clin Oncol. 2020;17(3):147-67.

8. Martinez M, Moon EK. CAR T cells for solid tumors: new strategies for finding, infiltrating, and surviving in the tumor microenvironment. Front Immunol. 2019;5(10): 128.

9. Depil S, Duchateau P, Grupp SA, Mufti G, Poirot L. 'Off-theshelf' allogeneic CAR T cells: development and challenges. Nat Rev Drug Discov. 2020;19(3):185-99.

10. Myers JA, Miller JS. Exploring the NK cell platform for cancer immunotherapy. Nat Rev Clin Oncol. 2021;18(2):85-100.

11. Iriguchi $\mathrm{S}$, Kaneko $\mathrm{S}$. Toward the development of true "offthe-shelf" synthetic T-cell immunotherapy. Cancer Sci. 2019;110(1):16-22.

Publisher's Note Springer Nature remains neutral with regard to jurisdictional claims in published maps and institutional affiliations. 